\title{
Adiponectin deficiency increases leukocyte- endothelium interactions via upregulation of endothelial cell adhesion molecules in vivo
}

\author{
Raogo Ouedraogo, ${ }^{1}$ Yulan Gong, ${ }^{2}$ Brett Berzins, ${ }^{2}$ Xiandong Wu, ${ }^{1}$ Kalyankar Mahadev, ${ }^{1}$ \\ Kelly Hough, ${ }^{1}$ Lawrence Chan, ${ }^{3}$ Barry J. Goldstein, ${ }^{1}$ and Rosario Scalia ${ }^{2}$ \\ 1Division of Endocrinology, Diabetes, and Metabolic Diseases, Department of Medicine, and 2Department of Molecular \\ Physiology and Biophysics, Jefferson Medical College, Thomas Jefferson University, Philadelphia, Pennsylvania, USA. \\ ${ }^{3}$ Section of Diabetes, Endocrinology, and Metabolism, Department of Medicine, Baylor College of Medicine, Houston, Texas, USA.
}

\begin{abstract}
This study reports on what we believe are novel mechanism(s) of the vascular protective action of adiponectin. We used intravital microscopy to measure leukocyte-endothelium interactions in adiponectin-deficient ( $\mathrm{Ad}^{-/-}$) mice and found that adiponectin deficiency was associated with a 2-fold increase in leukocyte rolling and a 5 -fold increase in leukocyte adhesion in the microcirculation. Measurement of endothelial NO (eNO) revealed that adiponectin deficiency drastically reduced levels of eNO in the vascular wall. Immunohistochemistry demonstrated increased expression of E-selectin and VCAM-1 in the vascular endothelium of $\mathrm{Ad}^{-/-}$mice. Systemic administration of the recombinant globular adiponectin domain (gAd) to $\mathrm{Ad}^{-/-}$mice significantly attenuated leukocyte-endothelium interactions and adhesion molecule expression in addition to restoring physiologic levels of eNO. Importantly, prior administration of gAd also protected WT mice against TNF- $\alpha$-induced leukocyte-endothelium interactions, indicating a pharmacologic action of gAd. Mechanistically, blockade of eNOS with $\mathbf{N}_{\omega}$-nitro-L-arginine methyl ester (L-NAME) abolished the inhibitory effect of gAd on leukocyte adhesion, demonstrating the obligatory role of eNOS signaling in the antiinflammatory action of gAd. We believe this is the first demonstration that gAd protects the vasculature in vivo via increased NO bioavailability with suppression of leukocyte-endothelium interactions. Overall, we provide evidence that loss of adiponectin induces a primary state of endothelial dysfunction with increased leukocyte-endothelium adhesiveness.
\end{abstract}

\section{Introduction}

Adiponectin is an adipocyte-derived protein with insulin-sensitizing, antiinflammatory, and antiatherogenic properties, abundantly found in the plasma in various oligomeric forms $(1,2)$. In general, decreased plasma levels of adiponectin have been associated with obesity, a well-known risk factor for cardiovascular disease (3), type 2 diabetes (4), and hypertension (5) although no study to date has implicated specific adiponectin isoforms in these conditions. Accordingly, plasma adiponectin levels are significantly lower in patients with coronary artery disease, further suggesting that physiologic levels of adiponectin are necessary to maintain the normal, noninflammatory phenotype of the vascular wall $(4,6)$. In agreement with these clinical observations, adiponectin-deficient $\left(\mathrm{Ad}^{-/-}\right)$ mice develop inflammatory-type vascular disorders in response to mechanical injury. Thus, severe neointimal thickening and increased proliferation of vascular smooth muscle cells occur in arteries of $\mathrm{Ad}^{-/-}$mice following external cuff injury (7). Notably, restoration of physiologic levels of adiponectin by recombinant adenovirus transduction in vivo was shown to attenuate neointimal proliferation in $\mathrm{Ad}^{-/-}$mice by reducing smooth muscle cell proliferation (8).

Recent data have linked the vascular protective role of adiponectin with preservation of endothelial cell function. Reduced

Nonstandard abbreviations used: $\mathrm{Ad}^{-/-}$, adiponectin-deficient (mouse); $\mathrm{Ad}^{+/}$ adiponectin-hemizygous (mouse); CAM, cell adhesion molecule; eNO, endothelial $\mathrm{NO}$; gAd, globular adiponectin domain; K-H, Krebs-Henseleit; L-NAME, $\mathrm{N}_{\omega}$-nitro-Larginine methyl ester.

Conflict of interest: The authors have declared that no conflict of interest exists. Citation for this article: J. Clin. Invest. 117:1718-1726 (2007). doi:10.1172/JCI29623. endothelial NO (eNO) availability at the vascular wall causes endothelial dysfunction (9), and the dysfunctional endothelium expresses a proinflammatory phenotype characterized by elevated expression of atherogenic cell adhesion molecules (CAMs) (10), in part due to loss of eNO (11). Upregulation of CAMs at the endothelial cell surface initiates pathologic leukocyte-endothelial cell interactions (12), which ultimately expose the vascular wall and surrounding tissues to the damaging action of activated leukocytes. Ouchi and coworkers (13) showed that endothelium-dependent relaxation is impaired in $\mathrm{Ad}^{-/-}$mice, suggesting that loss of adiponectin impairs eNO signaling. In addition, adiponectin has been shown to enhance NO production in cultured endothelial cells in vitro (14-16). Recent evidence has linked increased leukocyte-endothelium interactions in the microcirculation with inflammatory signal activation and the vascular complications of diabetes (17), a metabolic condition associated with reduced levels of adiponectin (4). Indeed, in vitro studies demonstrate that adiponectin exhibits antiinflammatory effects, including the suppression of TNF- $\alpha$-induced adhesion molecule expression (18).

In the present work, we sought to address the following: (a) the impact of adiponectin deficiency on baseline leukocyteendothelial cell interactions in the microcirculation in vivo; (b) the effect of administration of globular adiponectin domain (gAd) on leukocyte-endothelium interactions in $\mathrm{Ad}^{-/-}$mice as well as in WT mice exhibiting TNF- $\alpha$-induced vascular inflammation; and (c) the role of $\mathrm{NO}$ in the vascular protective action of adiponectin in vivo. Our results clearly demonstrate that loss of adiponectin function induces endothelial dysfunction characterized by loss of eNO and increased leukocyte-endothelium 

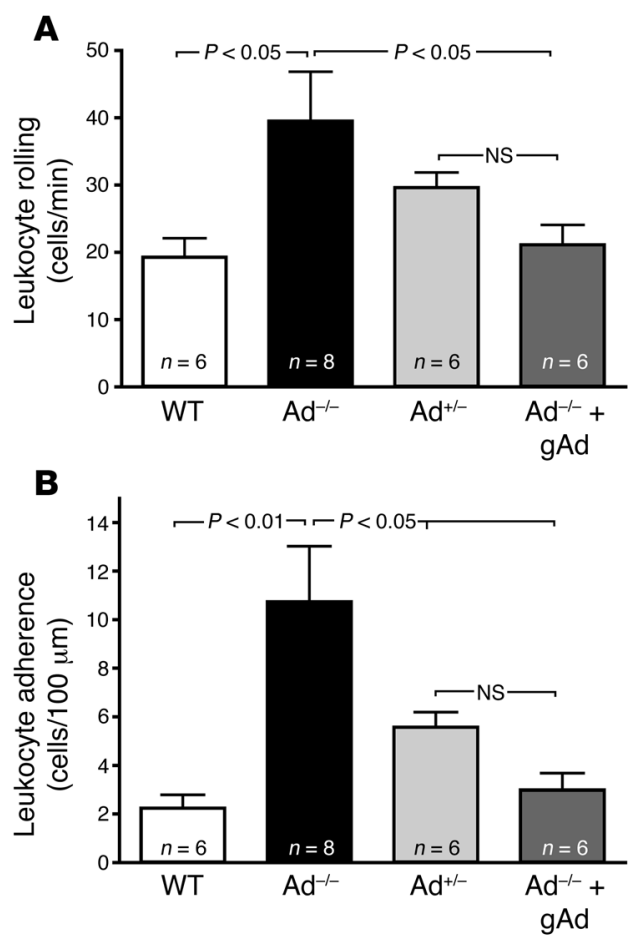

interactions. We also found that administration of gAd effectively preserves endothelial function in both acute inflammation and states of chronic adiponectin deficiency.

\section{Results}

Adiponectin deficiency increases leukocyte-endothelium interactions. To study the effect of adiponectin deficiency on vascular inflammation, we monitored basal leukocyte-endothelium interactions in WT mice, mice completely lacking adiponectin ( $\left.\mathrm{Ad}^{-/-}\right)$, and adiponectin-hemizygous $\left(\mathrm{Ad}^{+/-}\right)$mice. WT mice had endogenous total adiponectin plasma levels in the range of $11 \pm 0.8 \mu \mathrm{g} / \mathrm{ml}$. In contrast, levels of total adiponectin were virtually undetectable in the serum of $\mathrm{Ad}^{-1-}$ mice (average values were $0.1 \pm 0.1 \mu \mathrm{g} / \mathrm{ml} ; P<0.001$ versus WT), confirming the functional phenotype of the adiponectin knockout mouse model used in our study (19). Following administration of $25 \mu \mathrm{g}$ gAd s.c. to $\mathrm{Ad}^{-/-}$mice, serum gAd levels increased to $0.55 \pm 0.1 \mu \mathrm{g} / \mathrm{ml}(n=2)$. Ad $^{+/-}$mice obtained by extensive (>15 generations) backcrossing of Ad $/ /$ mice with WT C57BL/6J mice showed a $58 \%$ reduction in total plasma adiponectin levels compared with WT mice (average values were $4.6 \pm 1 \mu \mathrm{g} / \mathrm{ml}$; $P<0.01$ versus WT and $\mathrm{Ad}^{-/}$). Thus, unpairing of the adiponectin gene induces the expression of intermediate phenotypes.

Intravital microscopy studies revealed a very low number of rolling leukocytes in peri-intestinal venules of control WT mice (Figure 1A). In contrast, $\mathrm{Ad}^{-/-}$mice exhibited a 2-fold increase in the number of rolling leukocytes. Leukocyte rolling was also increased in $\mathrm{Ad}^{+/-}$mice although to a lower degree than in $\mathrm{Ad}^{-/}$mice (Figure 1A). Moreover, leukocyte rolling velocities $\left(31.04 \pm 1.50 \mu \mathrm{m} / \mathrm{s}\right.$ in WT mouse venules) were reduced in $\mathrm{Ad}^{+/-}$ and $\mathrm{Ad}^{-/}$mice, with the slowest leukocyte rolling velocity detected in $\mathrm{Ad}^{-/-}$mice $(19.40 \pm 0.44 \mu \mathrm{m} / \mathrm{s} ; P<0.01$ versus WT; Figure 2$)$. These data demonstrate that reduced levels of serum adiponectin are associated with progressively increased leukocyte-endothelium interactions in the microcirculation.

\section{Figure 1}

Leukocyte-endothelium interactions in mouse peri-intestinal venules are increased by endogenous adiponectin deficiency. (A) Leukocyte rolling flux in WT mice and $\mathrm{Ad}^{-/-}$mice before and after treatment with human recombinant gAd (25 $\mu$ g, s.c., twice daily for 10 days). (B) Leukocyte adhesion values under the same experimental conditions. Data are expressed as the mean \pm SEM. Numbers at the base of the bars indicate the number of mice studied in each group. Pharmacologically active doses of gAd prevent abnormal leukocyte-endothelial cell interactions in the microcirculation in the face of chronic adiponectin deficiency.

A very low number of leukocytes were also found to adhere to the vascular endothelium of WT mice (Figure 1B). Conversely, leukocyte adhesion increased 5-fold and 2.5-fold in $\mathrm{Ad}^{-/-}$mice and $\mathrm{Ad}^{+/-}$ mice ( $P<0.01$ and $P<0.05$ versus WT, respectively). As shown in Table 1 , no physiologically meaningful differences in body weight, blood glucose levels, mean arterial blood pressure, venular diameters, or total leukocyte counts were observed among the groups of mice studied. However, leukocyte blood counts increased after injection of the E-selectin blocking antibody 9A9, probably due to mobilization of adhering leukocytes to the circulating blood compartment (20). Thus, under our experimental conditions, changes in leukocyte-endothelium interactions were not due to alterations in glucose metabolism, circulating leukocyte numbers, systemic hemodynamic variables, or local rheologic factors.

Parallel experiments in isolated mouse aorta ex vivo demonstrated that the aortic endothelium of $\mathrm{Ad}^{-/-}$mice has significantly increased adhesiveness to formyl-Met-Leu-Phe-activated human monocytic cells. For these studies, 12 aortic segments from 3 mice were studied in each group. The average number of monocytic cells adhering to control aorta from WT mice was only $4.3 \pm 0.4$ cells per microscopic field. In contrast, a total of $10.1 \pm 0.6$ and $7.7 \pm 0.7$ cells per microscopic field adhered to aortic segments isolated from $\mathrm{Ad}^{-/-}$and $\mathrm{Ad}^{+/-}$mice, respectively $(P<0.01$ versus WT aorta). These data are in agreement with data on leukocyte adhesion obtained by intravital microscopy, and they demonstrate that in the setting of declining levels of adiponectin, the vascular endothelium develops a proadhesive phenotype widely affecting large conduit vessels as well as the microcirculation.

gAd treatment attenuates leukocyte-endothelium interactions. To ascertain whether restoration of adiponectin signaling could reverse the abnormalities in leukocyte-endothelium interactions observed in $\mathrm{Ad}^{-/-}$mice, we treated $\mathrm{Ad}^{-/-}$mice with human recombinant gAd for a period of 10 days, as described in Methods. Prior studies have shown that gAd exhibits a variety of salutary effects in the vasculature, including reduction of oxidative stress in cultured endothelial cells $(16,18,21)$, and atheroprotective effects in the cardiovascular system of the mouse, as recently reviewed by our group (22).

As measured by intravital microscopy, treatment with gAd normalized leukocyte rolling flux, leukocyte rolling velocity, and leukocyte adhesion to the values found in control WT mice (Figures 1 and 2). These data support the hypothesis that the vascular inflammation observed in states of adiponectin deficiency is amenable to treatment with selected adiponectin isoforms, specifically gAd.

gAd treatment reverses TNF- $\alpha$ induced leukocyte-endothelium interactions in WT mice. TNF- $\alpha$ is a circulating cytokine that induces endothelial dysfunction characterized by downregulation of the eNOS/NO biosynthetic machinery (23-25) and upregulation of endothelial CAM expression (11). Interestingly, adiponectin has been reported to reverse some of the in vitro inflammatory signaling effects of TNF- $\alpha$ 

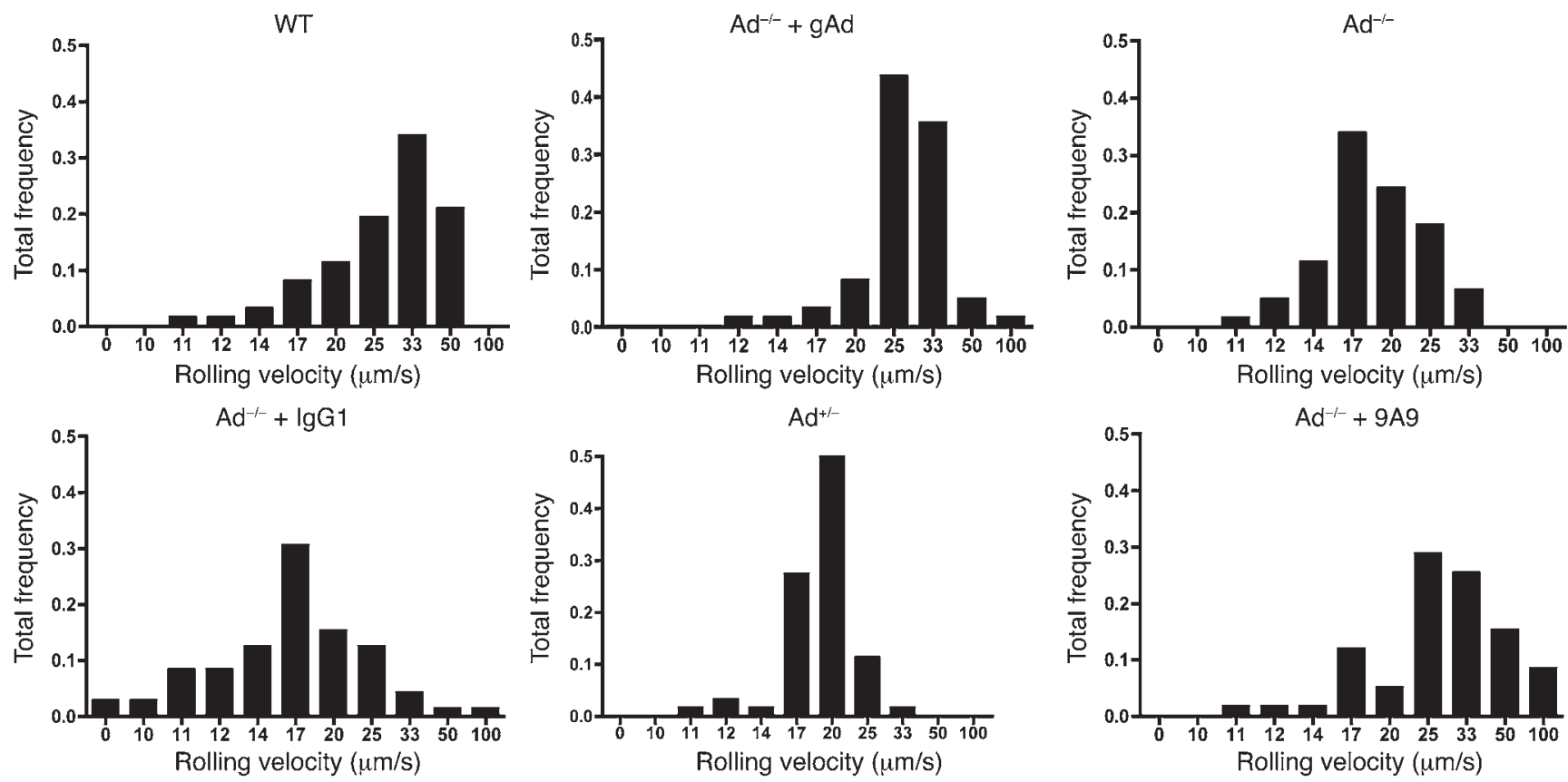

Figure 2

Endogenous adiponectin deficiency decreases leukocyte rolling velocity in the microcirculation. Panels illustrate rolling velocities in all experimental groups of mice. Data are expressed as the mean and represent the total leukocyte rolling frequency calculated from 5 mice in each group. Treatment of $\mathrm{Ad}^{-1-}$ mice with human recombinant gAd (25 $\mu$ g, s.c., twice daily for 10 days) normalizes leukocyte rolling velocity (top, middle panel). Similar results were obtained following functional blockade of E-selectin with the $\mathrm{mAb} 9 \mathrm{~A} 9 \mathrm{in} \mathrm{Ad}^{-/-}$mice $(2.1 \mathrm{mg} / \mathrm{kg}$, i.v.; bottom, right panel).

in cultured endothelial cells (6). Accordingly, we determined whether pharmacologically active doses of gAd could inhibit TNF- $\alpha$-mediated leukocyte-endothelium interactions in WT mice expressing physiological levels of adiponectin. Two-hour exposure of the mouse microcirculation to $1 \mu \mathrm{g} / \mathrm{kg}$ TNF- $\alpha$ given i.p. increased leukocyte rolling 3-fold $(P<0.01$; Figure $3 \mathrm{~A})$ and leukocyte adhesion 6-fold $(P<0.01$; Figure 3B). Treatment of mice with 2 consecutive doses of gAd ( $25 \mu \mathrm{g} /$ mouse, s.c.), given 24 hours and repeated 2.5 hours before TNF- $\alpha$ administration, attenuated TNF- $\alpha$-mediated leukocyte rolling by 2 -fold and leukocyte adhesion by 3 -fold $(P<0.01$; Figure 3 ). These data demonstrate that pharmacologically active doses of gAd dramatically protect the vasculature against cytokine-induced inflammation, even in the presence of endogenous adiponectin.
Endogenous adiponectin and gAd treatment regulates $N O$ availability in the vascular wall. To test the hypothesis that the antiinflammatory effect of adiponectin in the vasculature is accompanied by enhanced availability of eNO, we measured eNO release from the isolated mouse aorta. Adiponectin deficiency was associated with a $40 \%$ reduction in NO production compared with that of control WT mice (Table 2; $P<0.01$ versus WT aorta). Similarly, 2 hours after i.p. injection of $1 \mu \mathrm{g} / \mathrm{kg}$ of TNF- $\alpha$ into WT mice, eNO production in the aorta was reduced by $36 \%$ (Table $2 ; P<0.01$ versus control WT mice). Treatment of $\mathrm{Ad}^{-/-}$mice with gAd for 6 consecutive days restored eNO production significantly toward the level observed in WT mice (Table 2; $P>0.05$ versus WT). The effect of TNF- $\alpha$ to suppress eNO generation was also blocked by $55 \%$

Table 1

Main metabolic and hemodynamic parameters in all experimental groups of mice

\begin{tabular}{|c|c|c|c|c|c|c|}
\hline $\begin{array}{l}\text { Group } \\
(n)\end{array}$ & $\begin{array}{l}\text { Blood glucose } \\
\text { (mg/dl) }\end{array}$ & $\begin{array}{c}\text { MABP } \\
(\mathrm{mmHg})\end{array}$ & $\begin{array}{l}\text { Wall shear } \\
\text { rate }\left(\mathrm{s}^{-1}\right)\end{array}$ & $\begin{array}{l}\text { Average venule } \\
\text { diameter }(\mu \mathrm{m})\end{array}$ & $\begin{array}{l}\text { Body weight } \\
\text { (g) }\end{array}$ & $\begin{array}{l}\text { wbc } \\
\left(10^{6}\right)\end{array}$ \\
\hline WT (6) & $131 \pm 6$ & $86 \pm 4$ & $1745 \pm 214$ & $31.7 \pm 2.0$ & $31 \pm 1$ & $6.0 \pm 0.6$ \\
\hline WT + TNF- $\alpha(7)$ & $137 \pm 4$ & $90 \pm 4$ & $2129 \pm 233$ & $27.6 \pm 1.7$ & $26 \pm 1$ & $5.3 \pm 0.5$ \\
\hline WT + TNF- $\alpha+\operatorname{gAd}(5)$ & $119 \pm 6$ & $94 \pm 4$ & $2430 \pm 411$ & $29.3 \pm 1.3$ & $27 \pm 1$ & $5.7 \pm 0.3$ \\
\hline WT + L-NAME (7) & $121 \pm 3$ & $91 \pm 3$ & $2749 \pm 135$ & $28.3 \pm 0.8$ & $28 \pm 0$ & $6.3 \pm 1.2$ \\
\hline WT + L-NAME + gAd (4) & $111 \pm 5$ & $101 \pm 3$ & $2125 \pm 90$ & $27.3 \pm 0.9$ & $26 \pm 1$ & $5.1 \pm 0.3$ \\
\hline $\mathrm{Ad}^{+/-}(6)$ & $127 \pm 5$ & $87 \pm 2$ & $1830 \pm 170$ & $29.0 \pm 1.8$ & $28 \pm 2$ & $5.6 \pm 0.6$ \\
\hline $\mathrm{Ad}^{-/-}(8)$ & $137 \pm 5$ & $89 \pm 4$ & $2084 \pm 170$ & $25.8 \pm 0.8$ & $26 \pm 1$ & $5.4 \pm 0.7$ \\
\hline $\mathrm{Ad}^{-/-}+\operatorname{gAd}(6)$ & $126 \pm 6$ & $75 \pm 6$ & $1624 \pm 76$ & $32.8 \pm 1.6$ & $26 \pm 1$ & $5.7 \pm 0.5$ \\
\hline $\mathrm{Ad}^{-/-}+\mathrm{L}-\mathrm{NAME}+\mathrm{gAd}(4)$ & $107 \pm 6$ & $98 \pm 3$ & $2661 \pm 175$ & $32.5 \pm 0.6$ & $33 \pm 2$ & $6.3 \pm 1.4$ \\
\hline $\mathrm{Ad}^{-/-}+\lg \mathrm{G} 1(6)$ & $113 \pm 4$ & $92 \pm 4$ & $2234 \pm 88$ & $26.7 \pm 0.3$ & $29 \pm 1$ & $5.8 \pm 0.5$ \\
\hline $\mathrm{Ad}^{-/-}+\mathrm{mAb} 9 \mathrm{~A} 9(6)$ & $122 \pm 7$ & $94 \pm 2$ & $2453 \pm 112$ & $24.3 \pm 0.5$ & $28 \pm 1$ & $7.0 \pm 0.9$ \\
\hline
\end{tabular}

MABP, mean arterial blood pressure. 

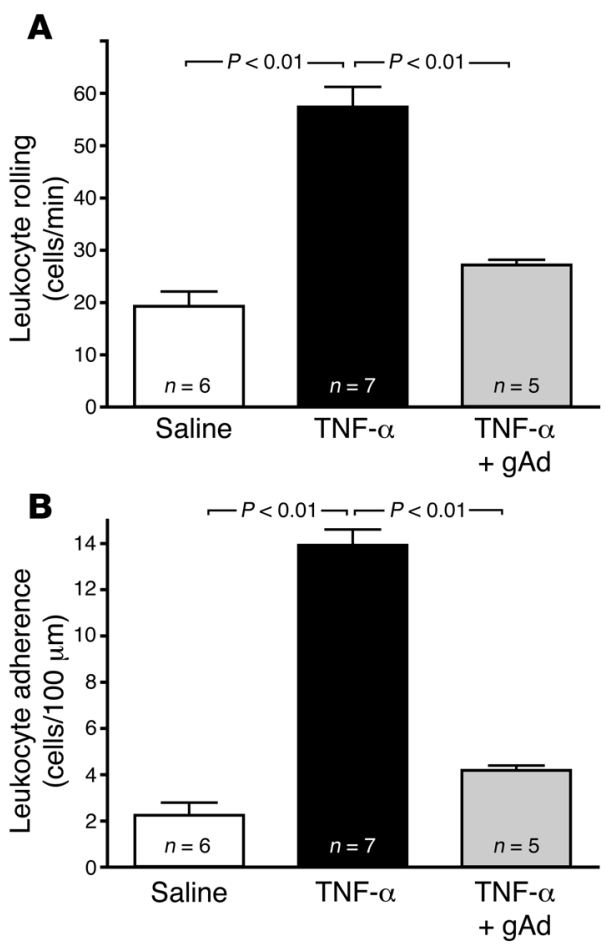

with prior treatment with a double dose of $25 \mu \mathrm{g}$ gAd, given as described above. These data clearly demonstrate that pharmacologically active doses of gAd increase NO bioavailability not only in states of adiponectin deficiency but also in the context of normal adiponectin levels when the cardiovascular system is exposed to elevated cytokine levels.

In vivo eNOS blockade suppresses the antiinflammatory action of $g A d$. To further substantiate the involvement of eNO availability in the vascular protective action of adiponectin, we blocked eNOS activity with the specific inhibitor $\mathrm{N}_{\omega}$-nitro-L-arginine methyl ester (L-NAME). In agreement with previous publications $(26,27)$, superfusion of the mesenteric microcirculation with $1 \mathrm{mM}$ L-NAME increased leukocyte adherence 5 -fold $(P<0.01$ versus control; Figure 4), thus producing an inflammatory signal comparable to that observed after injection of TNF- $\alpha$ (Figure 3). L-NAME-induced leukocyte adherence was not affected by systemic treatment of WT mice with recombinant gAd (Figure 4). Moreover, L-NAME superfusion abolished the inhibitory effect of gAd treatment on leukocyte adhesion in $\mathrm{Ad}^{-/-}$mice (Figure 4). These results demonstrate that functional eNOS activity is integrally involved in the antiinflammatory actions of gAd in the vasculature in vivo.

Endogenous adiponectin and $g$ Ad treatment regulate expression of endothelial CAMs: functional implications. To identify the underlying molecular mechanism(s) responsible for the increased leukocyteendothelium interactions observed in states of adiponectin deficiency, we used immunohistochemistry to measure expression levels of E-selectin and VCAM-1, 2 proinflammatory endothelial CAMs known to regulate leukocyte halting and leukocyte firm adhesion in the microcirculation. The percentage of venules staining positively for E-selectin in ileal sections from WT mice was consistently low (Figure 5). However, $\mathrm{Ad}^{-1-}$ and $\mathrm{Ad}^{+/-}$mice exhibited a significantly increased expression of E-selectin as quantified by the percentage of positively staining venules.

\section{Figure 3}

Pharmacologically active doses of gAd reverse TNF- $\alpha$-induced leukocyte-endothelium interactions in WT mice. As shown in A and B, $1 \mu \mathrm{g} / \mathrm{kg}$ TNF- $\alpha$ given 2 hours before the experiment increases leukocyte rolling flux and leukocyte adhesion, respectively, in the microcirculation of WT mice. Two $25 \mu \mathrm{g}$ doses of gAd given 24 hours and 2.5 hours before intravital microscopy significantly attenuates TNF- $\alpha$-induced leukocyte rolling and adhesion, confirming the potent antiinflammatory action of pharmacologic doses of gAd also in mice expressing physiologic adiponectin levels. Data are expressed as the mean \pm SEM. Numbers at the base of the bars indicate the number of mice studied in each group.

Increased E-selectin expression on the venular endothelium of $\mathrm{Ad}^{-/-}$mice was attenuated by gAd treatment (Figure 5). Similarly, few ileal venules stained positive for VCAM-1 in WT control mice (Figure 5). The number of ileal venules staining positive for VCAM-1 expression increased in $\mathrm{Ad}^{+/-}$mice and $\mathrm{Ad}^{-/-}$mice. Furthermore, treatment with gAd attenuated the expression of VCAM-1 in ileal venules of $\mathrm{Ad}^{-/-}$mice (Figure 5).

Previous studies have implicated E-selectin in both leukocyte rolling and leukocyte adhesion $(28,29)$. Accordingly, we sought to investigate the contribution of E-selectin to leukocyte adhesion in our $\mathrm{Ad}^{-/}$mouse model by acutely blocking E-selectin function with a bolus injection of the E-selectin mAb 9A9 to $\mathrm{Ad}^{-/-}$mice. Following systemic administration of the mAb 9A9, leukocyte adhesion in $\mathrm{Ad}^{-/-}$ mice was drastically reduced (Figure 6A), demonstrating that E-selectin is largely responsible for leukocyte adhesion in states of adiponectin deficiency. Leukocyte rolling values in $\mathrm{Ad}^{-/-}$were slightly increased following administration of mAb 9A9 although they failed to reach statistical significance (Figure 6B). Nonetheless, treatment with mAb 9A9 significantly increased leukocyte rolling velocity in $\mathrm{Ad}^{-/-}$mice from $19.3 \pm 1.4 \mu \mathrm{m} / \mathrm{s}$ to $35.6 \pm 2.9 \mu \mathrm{m} / \mathrm{s}$ (Figure 2). Together, these data strongly correlate with the functional changes in leukocyte-endothelium interactions shown in Figures 1 and 2. They also emphasize the role of E-selectin in the vascular inflammation associated with adiponectin-deficient states.

\section{Discussion}

This study was undertaken to investigate the early mechanisms of endothelial dysfunction associated with systemic adiponectin deficiency, an area of growing interest given the high incidence of vascular disease in metabolic states associated with obesity and

\section{Table 2}

Release of NO

$\begin{array}{lc}\text { Group }(\boldsymbol{n}) & \text { NO (pmol/mg tissue) } \\ \text { WT (3) } & 142.4 \pm 6.3 \\ \text { WT + TNF- } \alpha(3) & 90.7 \pm 7.8^{\mathrm{A}} \\ \text { WT }+ \text { gAd + TNF- } \alpha(3) & 119.2 \pm 6.9^{\mathrm{B}} \\ \mathrm{Ad}^{-/-}(3) & 84.8 \pm 4.7^{\mathrm{A}} \\ \mathrm{Ad}^{-/-}+\operatorname{gAd}(3) & 120.2 \pm 7.9^{\mathrm{C}}\end{array}$

NO release was measured in isolated mouse aortic rings obtained from WT or $\mathrm{Ad}^{-1-}$ mice given $0.9 \%$ saline or TNF- $\alpha$ i.p. $(1 \mu \mathrm{g} / \mathrm{kg}$, injected 2 hours before isolation of aortas). In the gAd-treated WT mouse group, mice were injected s.c. with $25 \mu \mathrm{g}$ of $\mathrm{gAd} /$ mouse 24 hours before and again 2.5 hours before isolation of aortic rings. In the $\mathrm{gAd}$-treated $\mathrm{Ad}^{-1-}$ mouse group, mice were injected s.c. with $25 \mu \mathrm{g}$ of $\mathrm{gAd} /$ mouse given twice a day for 6 consecutive days. All values were obtained from 3 mice in each group. Three aortic segments were studied in each mouse. ${ }^{A} P<0.01$ versus $W T$; ${ }^{\mathrm{B} P}<0.05$ versus WT + TNF- $\alpha ; \mathrm{C} P<0.05$ versus $\mathrm{Ad}^{-1-}$. 


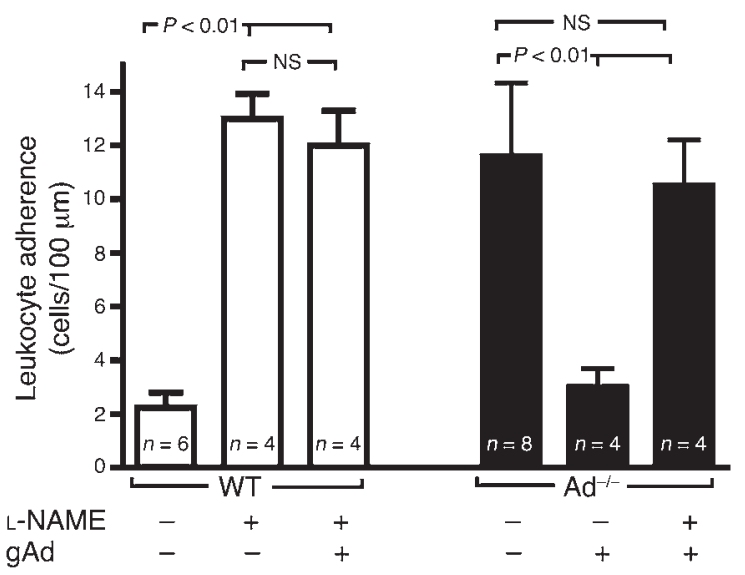

adipocyte dysfunction. We believe our results are the first in vivo demonstration that global adiponectin deficiency increases leukocyte-endothelial cell interactions via reduced availability of eNO at the vascular wall and upregulation of endothelial CAMs that are relevant to vascular inflammation and atherosclerosis. We also provide evidence that systemic administration of pharmacologically active doses of the recombinant gAd fragment alone reverts the endothelial dysfunction associated with adiponectin deficiency and attenuates cytokine-induced vascular inflammation in WT mice expressing physiologic concentrations of adiponectin in the blood.

Recruitment of circulating leukocytes is important in the pathophysiology of macrovascular (30) and microvascular diseases (17). The healthy endothelium does not bind circulating leukocytes under normal conditions. In the early stages of endothelial dysfunction, there is an increase in endothelial cell expression of various adhesion molecules on the luminal surface, including selectins and CAMs, all of which act as receptors for glycoconjugates and integrins present on the circulating leukocytes (31). Trafficking leukocytes are known to be detrimental to the vessel wall and tissues (32). Traditionally, it has been believed that prolonged exposure of the vascular endothelium to elevated circulating levels of metabolites or inflammatory mediators, such as glucose, free fatty acids, oxidized LDL, and cytokines, causes endothelial dysfunction by perturbing endothelial cell homeostasis. Recent research, however, has emphasized the role of adipose tissue and the deranged secretory properties of adipocytes in obesity as major causes of endothelial dysfunction (2). Dysfunction of adipose tissue, such as that occurring in obesity and insulin resistance, is characterized by systemic activation of inflammatory signals (33). Many of these signals arise directly or indirectly from secretory products of white adipocytes and actively contribute to the circulating milieu that induces vascular dysfunction $(34,35)$.

The reduction of circulating adiponectin levels in obesity and insulin resistance $(4,36)$ is of particular interest in view of the fact that low adiponectin levels are associated with endothelial dysfunction in humans $(14,37,38)$. This observation has been confirmed in several relevant animal models of disease in which reduced blood levels of adiponectin have been proven to exacerbate vascular injury $(8,39,40)$. In vivo studies have also proven that

\section{Figure 4}

Functional eNOS activity is necessary for the antiinflammatory effect of gAd. Superfusion of the mouse mesenteric microcirculation with the eNOS inhibitor L-NAME increases leukocyte adhesion in WT mice (white bars). Two $25 \mu \mathrm{g}$ doses of gAd given 24 hours and 2.5 hours before intravital microscopy failed to prevent leukocyte adhesion in response to L-NAME. Moreover, attenuation of leukocyte adhesion by administration of gAd $(25 \mu \mathrm{g} /$ twice daily for 6 consecutive days) to $\mathrm{Ad}^{-/-}$mice is acutely hampered by superfusion of the microcirculation with L-NAME (black bars). Data are expressed as the mean \pm SEM. Numbers at the base of the bars indicate the number of mice studied in each group.

adiponectin overexpression protects ApoE-deficient mice from atherosclerosis (41). Evidence has been also published to show that circulating adiponectin binds to the vascular wall of catheter-injured vessels, although the functional implications of this observation for vascular homeostasis are not known (42). More mechanistic studies in vitro have demonstrated that this adipokine is provided with antiinflammatory properties against oxidative stress and the action of cytokines $(18,43)$, suggesting that inhibition of inflammatory signals is the fundamental mechanism of the vascular protective actions of adiponectin in vivo.

Our data demonstrating that adiponectin deficiency increases leukocyte rolling and leukocyte adhesion help to explain a key mechanism by which loss of physiologic levels of adiponectin causes a generalized state of chronic vascular inflammation in vivo.
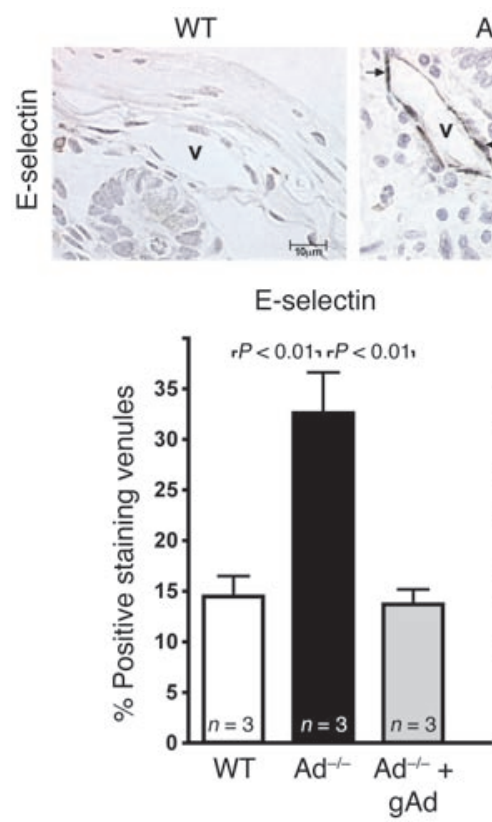

$\mathrm{Ad}^{-1-}$

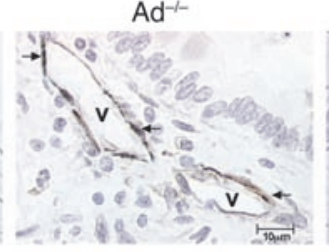

$\mathrm{Ad}^{-1-}+\mathrm{gAd}$

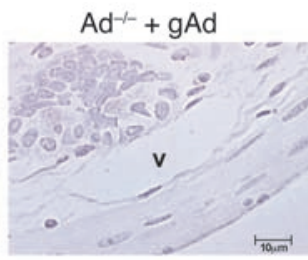

VCAM-1

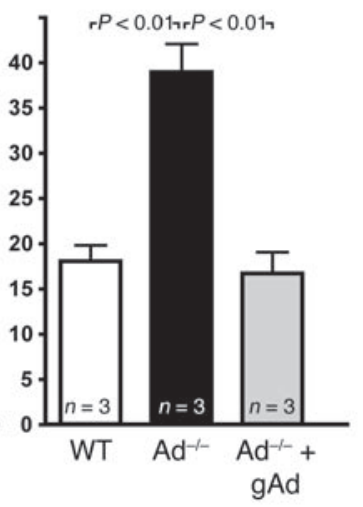

\section{Figure 5}

Adiponectin deficiency increases E-selectin and VCAM-1 expression levels in the vascular endothelium of the mouse microcirculation. Representative sections of ileal venules (Vs) staining positive for E-selectin are shown in the top 3 photomicrographs. E-selectin staining in all experimental groups of mice was detected by avidin/biotin immunoperoxidase technique seen as brown reaction product (arrows). Original magnification, $\times 500$. Bottom graphs show quantification of the percentage of venules staining positive for E-selectin (left) and VCAM-1 (right). Values are mean \pm SEM. Numbers at base of bars indicate the number of mice studied in each group. Five tissue sections were studied in each mouse, and 50 venules were analyzed per section. 

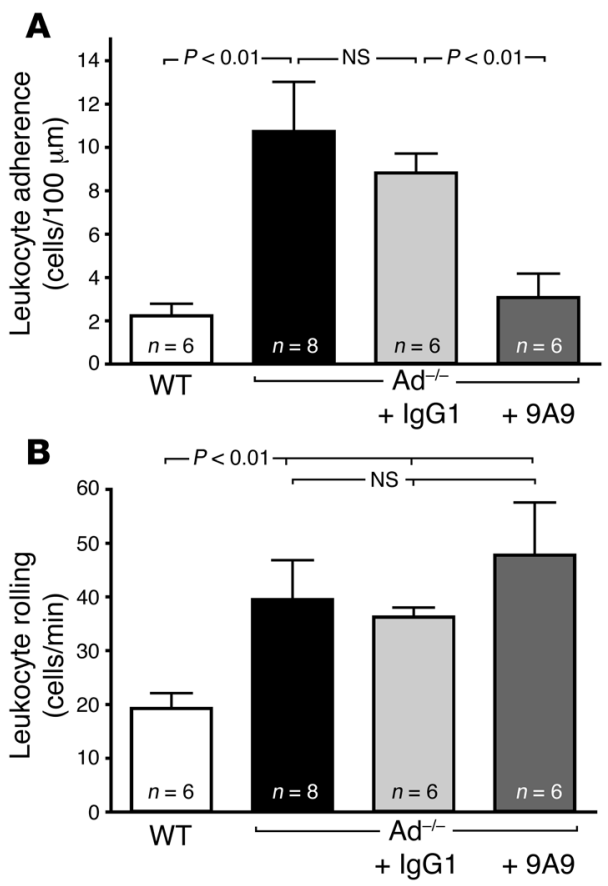

Specific adhesion molecules expressed on endothelial cells orchestrate the recruitment of circulating leukocytes under physiologic and pathophysiologic conditions. We found evidence of increased leukocyte rolling flux associated with reduced leukocyte rolling velocities and increased leukocyte adhesion (Figures 1 and 2) in the microcirculation of $\mathrm{Ad}^{-/-}$mice. Accordingly, we measured the expression of E-selectin and VCAM-1 in the $\mathrm{Ad}^{-/-}$vascular endothelium and found evidence of increased E-selectin and VCAM-1 expression in $\mathrm{Ad}^{-/}$mice. Upregulation of E-selectin reduces rolling velocities of leukocytes (20) and participates in the conversion of rolling to firm adhesion, as demonstrated by the fact that E-selectin-deficient mice have a reduced number of firmly adherent leukocytes in response to local chemoattractant (28) or cytokine stimulation (29). To determine whether the increased adhesion of leukocytes seen in $\mathrm{Ad}^{-/}$mice is functionally dependent on E-selectin, we blocked E-selectin function in vivo with a mAb. Consistent with the previous studies cited above, we found that blockade of E-selectin function in $\mathrm{Ad}^{-/}$- acutely increased leukocyte rolling velocity and inhibited leukocyte adhesion in the face of adiponectin deficiency. Taken together, these data highlight a role for E-selectin in the endothelial dysfunction and vascular inflammation associated with loss of physiologic levels of adiponectin.

The type I transmembrane glycoprotein VCAM-1 was also found to be upregulated in the vascular endothelium of mice deficient in adiponectin. VCAM-1 expression is very low or absent under physiologic conditions, and its expression levels can be rapidly induced by cytokines, such as TNF- $\alpha$ (31). VCAM-1 has been implicated in the process of atherogenesis and plaque formation both in the mouse and in humans $(44,45)$. Relevant to our study, VCAM-1 mediates firm adhesion of leukocytes by functioning as a counterreceptor to leukocyte integrins (46). Our functional studies demonstrated that leukocyte adhesion in the microcirculation of $\mathrm{Ad}^{-/}$ mice is largely dependent on E-selectin. Thus, future research must be undertaken to clarify whether VCAM-1 also plays a significant role in the vascular inflammation associated with adiponectin

\section{Figure 6}

E-selectin supports leukocyte adhesion in the $\mathrm{Ad}^{-/-}$microcirculation. (A) Leukocyte adhesion in $\mathrm{Ad}^{-/-}$mice before and after in vivo blockade of E-selectin with the E-selectin function-blocking mAb $9 A 9$ $(2.1 \mathrm{mg} / \mathrm{kg})$. Leukocyte adhesion was not affected by systemic administration of control IgG1 (2.1 mg/kg). Leukocyte rolling values in $\mathrm{Ad}^{-/}$ were slightly increased following administration of mAb 9A9, although they failed to reach statistical significance $(\mathbf{B})$. Values represent the mean \pm SEM of rolling or adherent leukocytes. Numbers at the base of the bars indicate the number of mice studied for each group.

deficiency, since we clearly found evidence of increased VCAM-1 expression in this condition (Figure 5).

In addition to upregulating CAMs, the dysfunctional endothelium experiences loss of eNO (11). Loss of eNO contributes to hypertension and several concomitant alterations, including increased expression of endothelial adhesion molecules that underlie the early processes of atherosclerosis (13-15). Overall, there is a consensus in the literature that the antiinflammatory action of eNO is largely based on its inhibitory effect on leukocyte-endothelium interactions. At the molecular level, NO suppresses leukocyteendothelium interactions by inhibiting exocytosis of Weibel-Palade bodies and modulating the activity of NF- $\mathrm{KB}(11,47)$. Interestingly, induction of E-selectin by cytokines occurs exclusively at the transcriptional level and requires proteasome-dependent activation of NF-KB for maximal expression (48). As with E-selectin, activation of the NF- $\mathrm{KB}$ signal transduction pathway is essential for cytokine-induced VCAM-1 expression (49). Adiponectin has been shown to inhibit NF-кB activation in endothelial cells (6). Here, we report that loss of circulating levels of adiponectin is associated with reduced bioavailability of eNO. Thus, our results clearly show that the presence of adiponectin in the mouse circulation contributes to maintaining the endogenous production of eNO. Consistent with these results, our laboratory and others have reported that adiponectin enhances NO production in cultured endothelial cells (14-16).

Directly relevant to the clinical application of these data is our finding that pharmacologically active doses of gAd almost completely reverse the increase in leukocyte-endothelium interactions observed in both $\mathrm{Ad}^{-/-}$mouse vasculature and WT mouse vasculature acutely inflamed with TNF- $\alpha$. Several adiponectin multimeric isoforms, in addition to full-length adiponectin, have been isolated in the blood and tissues of humans and laboratory animals $(1,2)$. Recent research has emphasized a role for the gAd fragment that is now known to have metabolic as well as vascular protective functions $(40,50)$. Indeed it has been hypothesized that gAd may circulate in the blood of humans at a very low concentration or processed from the full-length protein at a local site (50), although no definitive evidence has been provided. This fragment of adiponectin has also been hypothesized to be a pharmacologically potent form of adiponectin that may be generated by a proteolytic cleavage step in vivo, although work in this area is ongoing $(2,50)$. In support of a potential therapeutic role for gAd, however, our results show that gAd treatment affords significant pharmacological protection against vascular inflammation at concentrations well below those of the physiological levels of circulating full-length adiponectin complexes. At the molecular level, we found evidence that gAd attenuates expression levels of E-selectin and VCAM-1 in $\mathrm{Ad}^{-/-}$mice, a result that agrees with our functional observation demonstrating reduced leukocyte adhesion following gAd therapy in the mouse. 
Mechanistically, we also found evidence that gAd increases availability of eNO in $\mathrm{Ad}^{-/-}$mice and that blockade of eNOS enzyme activity with L-NAME abolishes the inhibitory effect of adiponectin on leukocyte endothelium interactions. Indeed, in TNF- $\alpha$-treated mice, gAd increased aortic eNO levels by approximately $50 \%$ while leukocyte-endothelial cell interaction in the mesenteric microcirculation was almost completely blocked. The apparent discrepancy between the antiinflammatory effect of gAd in the microcirculation and its eNO augmenting action in the aorta could be due in part to different sensitivities of microvascular and macrovascular endothelia to the NO potentiating action of gAd. Future studies on gAd receptor distribution and activity in different vasculatures are needed to fully understand the impact of adiponectin in the homeostasis of individual vascular beds. Taken together, these data clearly demonstrate that an eNO-related mechanism is involved in the vascular protective action of adiponectin.

The antiinflammatory action of gAd was also confirmed in the WT mouse vasculature inflamed with a clinically relevant cytokine such as TNF- $\alpha$. In fact, TNF- $\alpha / N F-\kappa B$ signaling is well recognized as a strong regulator of inflammation in the vasculature in humans (51). Relevant to this view, cytokines such as TNF- $\alpha$ are closely linked to the endothelial production of ROS (superoxide and $\mathrm{H}_{2} \mathrm{O}_{2}$ ), a central component of the inflammatory milieu that contributes to atherogenesis in some pathologic conditions $(52-54)$. ROS can quickly quench NO, a process that results in the formation of peroxynitrite, which has also been postulated to alter the catalytic activity of eNOS, diverting its synthesis from $\mathrm{NO}$ toward increased superoxide production (55). In addition, TNF- $\alpha$ directly downregulates basal and insulin-stimulated eNOs expression (56). Recent work has shown in vitro that gAd stimulation of NO generation is associated with enhanced eNOS complex formation with HSP90 (57). In agreement with this view, a recent study in vitro has demonstrated that full-length adiponectin suppresses TNF- $\alpha$-induced adhesion molecule expression in cultured endothelial cells (18). We have also previously shown suppression of ROS by gAd in cultured endothelial cells treated with oxidized LDL or high glucose $(16,28)$. However, additional studies are needed to further elucidate the potential pharmacological effects of various adiponectin isoforms, including multimers and adiponectin fragments such as gAd in vascular inflammation.

In summary, our data demonstrate a clear role for physiologic levels of adiponectin in the regulation of eNO bioavailability and leukocyte-endothelium interactions in vivo. They also demonstrate that the abnormal endothelial dysfunction occurring in pathologic conditions associated with adiponectin deficiency is amenable to gAd pharmacologic treatment.

\section{Methods}

Animal models and study protocols. This study was performed in accordance with NIH guidelines for the use of experimental animals. The Institutional Animal Care and Use Committee of Thomas Jefferson University approved all of the animal protocols. WT C57BL/6J mice were purchased from Charles River Laboratories. Ad ${ }^{-/-}$mice, extensively backcrossed to C57BL/6J controls, were generated and characterized as reported (19). $\mathrm{Ad}^{+/-}$mice were obtained by backcrossing $\mathrm{Ad}^{-/-}$with WT mice. In all reported experiments, male mice were studied at age 12-17 weeks. The role of adiponectin deficiency and gAd therapy on leukocyte-endothelium interactions was studied in the following 4 groups of mice: (a) WT mice given saline; (b) $\mathrm{Ad}^{-/-}$mice given saline; (c) $\mathrm{Ad}^{+/-}$mice given saline; and (d) $\mathrm{Ad}^{-/-}$mice given gAd of $25 \mu \mathrm{g}$ twice daily for 10 consecutive days. The inhibitory action of
gAd ( $25 \mu \mathrm{g}$ given 24 hours and repeated 2.5 hours before experiment) on leukocyte-endothelium interactions was also studied in WT mice injected with $1 \mu \mathrm{g} / \mathrm{kg}$ TNF- $\alpha$ i.p. 2 hours before experiment. In parallel experiments, the microcirculation of WT and $\mathrm{Ad}^{-/-}$mice was superfused with $1 \mathrm{mM}$ L-NAME to study the role of eNOS activity in the mechanism by which gAd inhibits leukocyte-endothelium interactions. L-NAME has been used before to induce leukocyte-endothelium interactions in the microcirculation (12). In additional studies, $\mathrm{Ad}^{-/-}$mice were given $2 \mathrm{mg} / \mathrm{kg}$ i.v. of the E-selectin function-blocking antibody $9 \mathrm{~A} 9$ or control IgG1 to determine the contribution of E-selectin to the increased leukocyte adhesion seen in states of adiponectin deficiency. Previous studies have demonstrated that mAb 9A9 efficiently blocks E-selectin function in the mouse $(28,58)$. In all experiments, pharmacologically active doses of gAd were delivered to the mouse via s.c. injection. Previous studies have shown that adiponectin injected s.c. is absorbed into the systemic circulation (59).

Fluorescence intravital microscopy of mouse peri-intestinal venules. Mice were anesthetized by i.p. injection of $70 \mathrm{mg} / \mathrm{kg}$ sodium pentobarbital. Additional sodium pentobarbital was administered i.v. as needed during the intravital microscopy observation time. Polyethylene catheters were inserted into the left carotid artery to monitor mean arterial blood pressure and into the right jugular vein for administration of saline, pentobarbital, rhodamine, and antibodies. A loop of ileal mesentery was exteriorized through a midline incision in the abdominal wall and placed in a temperature-controlled, fluid-filled Plexiglas chamber (developed in our laboratory) for observation of the peri-intestinal microcirculation by intravital microscopy. The ileum and mesenteric tissue were superfused throughout the experiment with a modified Krebs-Henseleit (K-H) buffer warmed to $37^{\circ} \mathrm{C}$ and bubbled with $95 \% \mathrm{~N}_{2} / 5 \% \mathrm{CO}_{2}$. Centerline red blood cell velocity $\left(V_{r b c}\right)$ was monitored in bright field using an optical Doppler velocimeter (Department of Systems Biology and Translational Medicine, Texas A\&M University System Health Science Center). $V_{r b c}$ and venular diameter $(D)$ were used to calculate the venular wall shear rate $(\gamma)$ with the following formula: $\gamma=4.9 \times 8\left(V_{\text {mean }} / D\right)$, where mean velocity of red blood cells $\left(V_{\text {mean }}\right)$ equals $V_{r b c} / 1.6(60)$.

Postcapillary venules (20- to $40-\mu \mathrm{m}$ diameter) were observed under fluorescence microscopy, using a previously described intravital microscopy unit (61). Leukocytes were fluorescently labeled in vivo by injection of $0.3 \mathrm{mg} / \mathrm{kg}$ Rhodamine 6G (Sigma-Aldrich) injected via the right jugular vein. Rhodamine 6G-labeled leukocytes were monitored at a final magnification of $\times 400$ using appropriate filter sets. The microscopic images were recorded on an IBM computer equipped with Image-Pro software (version 4.5; MediaCybernetics). Leukocytes were classified according to the quality or duration of their interaction with the venular wall as rolling or adherent. The leukocyte rolling flux was defined as the total number of leukocytes crossing the $100-\mu \mathrm{m}$ venular segment in 60 seconds at a velocity that was significantly lower than the centerline velocity. Leukocyte rolling velocity was determined by measuring the time required for a leukocyte to roll along a $100-\mu \mathrm{m}$ length of venule. Leukocytes that were stationary for more than 30 seconds were defined as adherent. Leukocyte adhesion is expressed as the number of cells $/ 100 \mu \mathrm{m}$ of venular length. For experiments with L-NAME, mice were allowed to stabilize for 30 minutes following surgery. Following stabilization, a 20 - to $40-\mu \mathrm{m}$-diameter postcapillary venule was chosen for observation. A baseline recording was made to establish basal values for leukocyte rolling and adherence. The mesentery was then superfused with $1 \mathrm{mM}$ L-NAME dissolved in K-H solution for 60 minutes. Video recordings were made at $0,15,30,45$, and 60 minutes after initiation of superfusion for quantification of leukocyte rolling and adherence.

Adhesion of cultured monocytes to the mouse aorta in vitro. For these studies, we used an in vitro adhesion assay previously described (62). In brief, the thoracic aorta was isolated from donor WT and $\mathrm{Ad}^{-/-}$mice euthanized with an overdose of sodium pentobarbital $(150 \mathrm{mg} / \mathrm{kg})$ injected i.p. The aorta 
was quickly removed and placed in cold, oxygenated PBS. After carefully removing the adventitia, aortas were cut to $4-\mathrm{mm}$ segments. The segments were then carefully opened longitudinally and placed into culture dishes containing $1 \mathrm{ml} \mathrm{K-H}$ solution with their luminal surfaces facing up.

Human monocytic cells (THP-1) were cultured in RPMI-16 (+2 mM glutamine $+0.05 \mathrm{mM} 2$ mercaptoethanol $+10 \%$ FBS; Invitrogen). Before adhesion assay, THP-1 cells were fluorescently labeled using Zynaxis PKH2 staining kit (Zynaxis Cell Sciences Inc.) according to a previously described method (63). All steps were performed at $25^{\circ} \mathrm{C}$. A suspension of THP-1 monocytic cells was incubated with $4 \mu \mathrm{M}$ PKH-2GL for a labeling period of 5 minutes. The labeling was stopped by adding PBS containing 10\% FCS. Monocytic cells were incubated with $100 \mathrm{nM}$ formyl-Met-Leu-Phe for 5 minutes. Activated monocytic cells were then incubated with the aortic segments at a concentration of $10^{5}$ cells/well for 20 minutes at room temperature in an orbital shaker platform (Scienceware; Bel-Art Products). The aortic segments were then removed, gently washed in fresh K-H solution, and placed lumen side up on a microscope slide. The aortic tissue was covered with a drop of immersion oil followed by a glass coverslip. The number of monocytic cells adhering to the endothelial surface in 4 separate microscopic fields was counted under epifluorescent microscopy at a magnification of $\times 200$. Results are expressed as total number of cells/microscopic field.

Measurement of NO production in isolated aorta. Basal level of NO was measured in WT mice, WT mice injected with TNF- $\alpha$, and $\mathrm{Ad}^{-/-}$mice. The effect of gAd on basal NO levels was also studied. Mouse thoracic aorta rings, freshly isolated from donor mice, as described above, were used as the source of primary endothelial cells for measurement of NO production. The thoracic aortas were cut into 4-mm rings, which were then opened and fixed by small pins with the endothelial surface facing upwards in 24-well culture dishes containing $1 \mathrm{ml}$ of prewarmed $\mathrm{K}-\mathrm{H}$ solution. After equilibration at $37^{\circ} \mathrm{C}$, $\mathrm{NO}$ released into the $\mathrm{K}-\mathrm{H}$ solution was measured as previously reported (64). Briefly, a polarographic NO electrode internally shielded to minimize external electrical interference was connected to NO meter (Apollo 4000; World Precision Instruments). Calibration of the NO electrode was performed daily prior to each experimental protocol as previously described (65). Standard calibration curve was obtained by graded final concentrations of $\mathrm{KNO}_{2}$ from 0 to $500 \mathrm{nM}$ into a calibration solution containing $0.1 \mathrm{M}$ potassium iodide and $0.1 \mathrm{M}$ $\mathrm{H}_{2} \mathrm{SO}_{4}$. Release of $\mathrm{NO}$ was expressed as pmol/mg of dry tissue.

Immunohistochemistry. Following intravital microscopy experiments, the thorax was carefully opened, avoiding excessive bleeding. The vasculature was then flushed to remove red blood cells by carefully injecting saline in the left ventricle while the right ventricle was cut open for drainage (66). Once the vasculature was free of red blood cells, mice were perfused with icecold $4 \%$ paraformaldehyde for 3-5 minutes. Sections of ileum $(3-4 \mathrm{~cm})$ were then collected and placed in $4 \%$ paraformaldehyde for 2 hours on ice. The tissue was then cut into rings, dehydrated by using graded acetone washes, and embedded in plastic (Immunobed; Polysciences Inc.). Sections $(5-\mu \mathrm{m}$ thick) were cut and transferred to Vectabond-coated slides (Vector Laboratories). Immunohistochemical localization of E-selectin and VCAM-1 was accomplished by using the avidin/ biotin immunoperoxidase technique (VECTASTAIN ABC Reagent: Vector Laboratories) and with mAbs against either E-selectin or VCAM-1, as described previously (67). Briefly, blocking serum (horse) was applied to the tissue for 30 minutes to reduce nonspecific binding, and then the tissue sections were incubated with the primary antibody at a dilution of $1 / 100$ for 24 hours. The tissue was then incubated with the biotinylated secondary antibody, and peroxidase staining was carried out using 3,3'-diaminobenzidine. Control preparations involved omission of the primary antibody or omission of the secondary antibody. A total of 5 to 6 sections were studied from each mouse. Fifty venules were then analyzed per tissue section, and the percentage of positive staining was determined.

Plasma measurements. Endogenous total adiponectin levels and levels of the injected gAd in mouse serum were determined using mouse -and human-specific immunoassay kits from R\&D Systems. A drop of blood was collected through tail vein puncture to measure blood glucose levels using an Accu-Chek handheld glucose meter (Roche Diagnostics).

Reagents and solutions. Recombinant gAd was purchased from PeproTech. Monoclonal antibodies to mouse E-selectin (10E9.6) and VCAM-1 (MR106) for immunohistochemistry were purchased from BD Biosciences. Isotype control for E-selectin antibody, rat IgG1, was purchased from BD Biosciences - Pharmingen. Human recombinant TNF- $\alpha$ was purchased from Sigma-Aldrich.

Statistics. All data are presented as mean \pm SEM for the number of determinations shown in the figure or table. All data on leukocyte rolling and adherence as well as on mean arterial blood pressure, shear rates, and NO production levels were compared by ANOVA with post hoc analysis by Neuman-Keuls corrected $t$ test. $P$ values of 0.05 or less were considered statistically significant.

\section{Acknowledgments}

This work was supported by NIH grants DK63018 and DK71360 (to B.J. Goldstein), NIH grant DK064344 (to R. Scalia), and NIH grants HL51586 and DK68037 (to L. Chan). The authors are very grateful to Barry Wolitzky for providing the mAb 9A9 and to Kevin J. Williams for providing the monocytic cell line THP-1 used in these studies.

Received for publication July 6, 2006, and accepted in revised form April 2, 2007.

Address correspondence to: Rosario Scalia, Department of Physiology, Jefferson Medical College, Thomas Jefferson University, 1020 Locust Street, Philadelphia, Pennsylvania 19107-6799, USA. Phone: (215) 503-6409; Fax: (215) 503-2073; E-mail: Rosario. Scalia@jefferson.edu. Or to: Barry J. Goldstein, Division of Endocrinology, Diabetes, and Metabolic Diseases, Jefferson Medical College, 1020 Locust Street, Philadelphia, Pennsylvania 191076799, USA. Phone: (215) 503-1272; Fax: (215) 923-7932; E-mail: Barry.Goldstein@jefferson.edu.
1. Goldstein, B.J., and Scalia, R. 2004. Adiponectin: a novel adipokine linking adipocytes and vascular function. J. Clin. Endocrinol. Metab. 89:2563-2568.

2. Scherer, P.E. 2006. Adipose tissue: from lipid storage compartment to endocrine organ. Diabetes. 55:1537-1545.

3. Arita, Y., et al. 1999. Paradoxical decrease of an adipose-specific protein, adiponectin, in obesity. Biochem. Biophys. Res. Commun. 257:79-83.

4. Hotta, K., et al. 2000. Plasma concentrations of a novel, adipose-specific protein, adiponectin, in type 2 diabetic patients. Arterioscler. Thromb. Vasc. Biol. 20:1595-1599.
5. Iwashima, Y., et al. 2004. Hypoadiponectinemia is an independent risk factor for hypertension. Hypertension. 43:1318-1323.

6. Ouchi, N., et al. 2000. Adiponectin, an adipocytederived plasma protein, inhibits endothelial NFkappaB signaling through a cAMP-dependent pathway. Circulation. 102:1296-1301.

7. Kubota, N., et al. 2002. Disruption of adiponectin causes insulin resistance and neointimal formation. J. Biol. Chem. 277:25863-25866.

8. Matsuda, M., et al. 2002. Role of adiponectin in preventing vascular stenosis. The missing link of adipo-vascular axis. J. Biol. Chem. 277:37487-37491.
9. Lefer, A.M., and Scalia, R. 2001. Nitric oxide in inflammation. In Physiology of inflammation. K. Ley, editor. Oxford University Press. New York, New York, USA. 447-472.

10. Kubes, P., Suzuki, M., and Granger, D.N. 1991. Nitric oxide: an endogenous modulator of leukocyte adhesion. Proc. Natl. Acad. Sci. U. S. A. 88:4651-4655.

11. De Caterina, R., et al. 1995. Nitric oxide decreases cytokine-induced endothelial activation. Nitric oxide selectively reduces endothelial expression of adhesion molecules and proinflammatory cytokines. J. Clin. Invest. 96:60-68. 
12. Davenpeck, K.L., Gauthier, T.W., and Lefer, A.M. 1994. Inhibition of endothelial-derived nitric oxide promotes P-selectin expression and actions in the rat microcirculation Gastroenterology. 107:1050-1058.

13. Ouchi, N., et al. 2003. Association of hypoadiponectinemia with impaired vasoreactivity. Hypertension. 42:231-234

14. Tan, K.C., et al. 2004. Hypoadiponectinemia is associated with impaired endothelium-dependent vasodilation. J. Clin. Endocrinol. Metab. 89:765-769.

15. Chen, H., Montagnani, M., Funahashi, T., Shimomura, I., and Quon, M.J. 2003. Adiponectin stimulates production of nitric oxide in vascular endothelial cells. J. Biol. Chem. 278:45021-45026.

16. Motoshima, H., Wu, X., Mahadev, K., and Goldstein, B.J. 2004. Adiponectin suppresses proliferation and superoxide generation and enhances eNOS activity in endothelial cells treated with oxidized LDL. Biochem. Biophys. Res. Commun. 315:264-271.

17. Joussen, A.M., et al. 2004. A central role for inflammation in the pathogenesis of diabetic retinopathy. FASEB J. 18:1450-1452.

18. Ouchi, N., et al. 1999. Novel modulator for endothelial adhesion molecules: adipocytederived plasma protein adiponectin. Circulation. 100:2473-2476.

19. Ma, K., et al. 2002. Increased beta-oxidation but no insulin resistance or glucose intolerance in mice lacking adiponectin. J. Biol. Chem 277:34658-34661.

20. Kunkel, E.J., and Ley, K. 1996. Distinct phenotype of E-selectin-deficient mice. E-selectin is required for slow leukocyte rolling in vivo. Circ. Res. 79:1196-1204.

21. Ouedraogo, R., et al. 2006. Adiponectin suppression of high-glucose-induced reactive oxygen species in vascular endothelial cells: evidence for involvement of a cAMP signaling pathway. Diabetes. 55:1840-1846.

22. Goldstein, B.J., and Scalia, R. 2007. Adipokines and vascular disease in diabetes. Curr. Diab. Rep. 7:25-33.

23. Valerio, A., et al. 2006. TNF- $\alpha$ downregulates eNOS expression and mitochondrial biogenesis in fat and muscle of obese rodents. J. Clin. Invest. 116:2791-2798. doi:10.1172/JCI28570.

24. Picchi, A., et al. 2006. Tumor necrosis factor-alpha induces endothelial dysfunction in the prediabetic metabolic syndrome. Circ. Res. 99:69-77.

25. Neumann, P., Gertzberg, N., and Johnson, A 2004. TNF-alpha induces a decrease in eNOS promoter activity. Am. J. Physiol. Lung Cell. Mol. Physiol. 286:L452-L459.

26. Pruefer, D., Scalia, R., and Lefer, A.M. 1999. Simvastatin inhibits leukocyte-endothelial cell interactions and protects against inflammatory processes in normocholesterolemic rats. Arterioscler. Thromb. Vasc. Biol. 19:2894-2900.

27. Lefer, D.J., et al. 1999. Leukocyte-endothelial cell interactions in nitric oxide synthase-deficient mice. Am. J. Physiol. 276:H1943-H1950.

28. Ley, K., Allietta, M., Bullard, D.C., and Morgan, S. 1998. Importance of E-selectin for firm leukocyte adhesion in vivo. Circ. Res. 83:287-294

29. Milstone, D.S., et al. 1998. Mice lacking E-selectin show normal numbers of rolling leukocytes but reduced leukocyte stable arrest on cytokine-activated microvascular endothelium. Microcirculation. 5:153-171.

30. Krieglstein, C.F., and Granger, D.N. 2001. Adhesion molecules and their role in vascular disease. Am. J. Hypertens. 14:44S-54S.

31. Carlos, T.M., and Harlan, J.M. 1994. Leukocyte- endothelial adhesion molecules. Blood. 84:2068-2101.

32. Weiss, S.J. 1989. Tissue destruction by neutrophils. N. Engl. J. Med. 320:365-376.

33. Festa, A., et al. 2001. The relation of body fat mass and distribution to markers of chronic inflammation. Int. J. Obes. Relat. Metab. Disord. 25:1407-1415.

34. Fruhbeck, G., Gomez-Ambrosi, J., Muruzabal, F.J., and Burrell, M.A. 2001. The adipocyte: a model for integration of endocrine and metabolic signaling in energy metabolism regulation. Am. J. Physiol. Endocrinol. Metab. 280:E827-E847.

35. Trayhurn, P., and Wood, I.S. 2004. Adipokines: inflammation and the pleiotropic role of white adipose tissue. Br. J. Nutr. 92:347-355.

36. Arita, Y., et al. 1999. Paradoxical decrease of an adipose-specific protein, adiponectin, in obesity. Biochem. Biophys. Res. Commun. 257:79-83.

37. Shimabukuro, M., et al. 2003. Hypoadiponectinemia is closely linked to endothelial dysfunction in man. J. Clin. Endocrinol. Metab. 88:3236-3240.

38. Fernandez-Real, J.M., et al. 2004. Adiponectin is associated with vascular function independent of insulin sensitivity. Diabetes Care. 27:739-745.

39. Kubota, N., et al. 2002. Disruption of adiponectin causes insulin resistance and neointimal formation. J. Biol. Chem. 277:25863-25866.

40. Yamauchi, T., et al. 2003. Globular adiponectin protected ob/ob mice from diabetes and ApoEdeficient mice from atherosclerosis. J. Biol. Chem. 278:2461-2468.

41. Okamoto, Y., et al. 2002. Adiponectin reduces atherosclerosis in apolipoprotein E-deficient mice. Circulation. 106:2767-2770.

42. Okamoto, Y., et al. 2000. An adipocyte-derived plasma protein, adiponectin, adheres to injured vascular walls. Horm. Metab. Res. 32:47-50.

43. Nakanishi, S., et al. 2005. A protective effect of adiponectin against oxidative stress in Japanese Americans: the association between adiponectin or leptin and urinary isoprostane. Metabolism. 54:194-199.

44. Nakashima, Y., Raines, E.W., Plump, A.S., Breslow, J.L., and Ross, R. 1998. Upregulation of VCAM-1 and ICAM-1 at atherosclerosis-prone sites on the endothelium in the ApoE-deficient mouse. Arterioscler. Thromb. Vasc. Biol. 18:842-851.

45. O’Brien, K.D., et al. 1993. Vascular cell adhesion molecule- 1 is expressed in human coronary atherosclerotic plaques. Implications for the mode of progression of advanced coronary atherosclerosis. J. Clin. Invest. 92:945-951.

46. Luscinskas, F.W., Ding, H., and Lichtman, A.H. 1995. P-selectin and vascular cell adhesion molecule 1 mediate rolling and arrest, respectively, of CD4+ $\mathrm{T}$ lymphocytes on tumor necrosis factor alpha-activated vascular endothelium under flow. J. Exp. Med. 181:1179-1186.

47. Matsushita, K., et al. 2003. Nitric oxide regulates exocytosis by S-nitrosylation of N-ethylmaleimidesensitive factor. Cell. 115:139-150.

48. Collins, T., et al. 1995. Transcriptional regulation of endothelial cell adhesion molecules: NF-kappa $\mathrm{B}$ and cytokine-inducible enhancers. FASEB J. 9:899-909.

49. Ghosh, S., May, M.J., and Kopp, E.B. 1998. NF-kappa $\mathrm{B}$ and Rel proteins: evolutionarily conserved mediators of immune responses. Annu. Rev. Immunol. 16:225-260.

50. Fruebis, J., et al. 2001. Proteolytic cleavage product of $30-\mathrm{kDa}$ adipocyte complement-related protein increases fatty acid oxidation in muscle and causes weight loss in mice. Proc. Natl. Acad. Sci. U. S. A. 98:2005-2010

51. Madge, L.A., and Pober, J.S. 2001. TNF signaling in vascular endothelial cells. Exp. Mol. Pathol.
70:317-325.

52. Fernandez-Real, J.M., and Ricart, W. 2003. Insulin resistance and chronic cardiovascular inflammatory syndrome. Endocr. Rev. 24:278-301.

53. Kuroki, T., Isshiki, K., and King, G.L. 2003. Oxidative stress: the lead or supporting actor in the pathogenesis of diabetic complications. J. Am. Soc. Nephrol. 14:S216-S220.

54. Singleton, J.R., Smith, A.G., Russell, J.W., and Feldman, E.L. 2003. Microvascular complications of impaired glucose tolerance. Diabetes. 52:2867-2873.

55. Zou, M.H., Shi, C., and Cohen, R.A. 2002. Oxidation of the zinc-thiolate complex and uncoupling of endothelial nitric oxide synthase by peroxynitrite. J. Clin. Invest. 109:817-826. doi:10.1172/JCI200214442.

56. Aljada, A., Ghanim, H., Assian, E., and Dandona, P. 2002. Tumor necrosis factor-alpha inhibits insulin-induced increase in endothelial nitric oxide synthase and reduces insulin receptor content and phosphorylation in human aortic endothelial cells. Metabolism. 51:487-491.

57. Xi, W., Satoh, H., Kase, H., Suzuki, K., and Hattori, Y. 2005. Stimulated HSP90 binding to eNOS and activation of the PI3-Akt pathway contribute to globular adiponectin-induced $\mathrm{NO}$ production: vasorelaxation in response to globular adiponectin. Biochem. Biophys. Res. Commun. 332:200-205.

58. Ramos, C.L., et al. 1997. Differential effect of E-selectin antibodies on neutrophil rolling and recruitment to inflammatory sites. Blood. 89:3009-3018

59. Wang, Y., et al. 2006. Post-translational modifications of the four conserved lysine residues within the collagenous domain of adiponectin are required for the formation of its high molecular weight oligomeric complex. J. Biol. Chem. 281:16391-16400.

60. Smith, M.L., Long, D.S., Damiano, E.R., and Ley, K. 2003. Near-wall micro-PIV reveals a hydrodynamically relevant endothelial surface layer in venules in vivo. Biophys. J. 85:637-645.

61. Scalia, R., Armstead, V.E., Minchenko, A.G., and Lefer, A.M. 1999. Essential role of P-selectin in the initiation of the inflammatory response induced by hemorrhage and reinfusion. J. Exp. Med. 189:931-938.

62. Lefer, D.J., et al. 1997. Peroxynitrite inhibits leukocyte-endothelial cell interactions and protects against ischemia-reperfusion injury in rats. J. Clin. Invest. 99:684-691.

63. Yuan, Y., and Fleming, B.P. 1990. A method for isolation and fluorescent labeling of rat neutrophils for intravital microvascular studies. Microvasc. Res. 40:218-229.

64. Booth, G., Stalker, T.J., Lefer, A.M., and Scalia, R. 2001. Elevated ambient glucose induces acute inflammatory events in the microvasculature: effects of insulin. Am. J. Physiol. Endocrinol. Metab. 280:E848-E856.

65. Guo, J.P., Murohara, T., Buerke, M., Scalia, R., and Lefer, A.M. 1996. Direct measurement of nitric oxide release from vascular endothelial cells. J. Appl. Physiol. 81:774-779.

66. Scalia, R., Gefen, J., Petasis, N.A., Serhan, C.N., and Lefer, A.M. 1997. Lipoxin A4 stable analogs inhibit leukocyte rolling and adherence in the rat mesenteric microvasculature: role of P-selectin. Proc. Natl. Acad. Sci. U. S. A. 94:9967-9972.

67. Weyrich, A.S., Buerke, M., Albertine, K.H., and Lefer, A.M. 1995. Time course of coronary vascular endothelial adhesion molecule expression during reperfusion of the ischemic feline myocardium. J. Leukoc. Biol. 57:45-55. 